\title{
ß-Glucan-Based Wet Dressing for Cutaneous Wound Healing
}

\author{
Karthika Muthuramalingam, ${ }^{1}$ Seung In $\mathrm{Choi}^{2}{ }^{2}$ Changlim Hyun, \\ Young Mee Kim, ${ }^{1, *}$ and Moonjae Cho ${ }^{1,4, *}$ \\ Departments of ${ }^{1}$ Biochemistry and ${ }^{3}$ Pathology, School of Medicine, Jeju National University, Jeju, Republic of Korea. \\ ${ }^{2}$ Department of Pharmaceutical Research Institute, Quegen Biotech Co. Ltd., Seoul, Republic of Korea. \\ ${ }^{4}$ Department of Biochemistry, School of Medicine, Institute of Medical Science, Jeju National University, \\ Jeju, Republic of Korea.
}

Objective: Recognized as pathogen-associated molecular patterns (PAMPs), $\beta$ glucans, a naturally occurring heterogeneous group of polysaccharides, were investigated for their ability to accelerate wound healing in the form of high water-retaining hydrogel dressing.

Approach: Full-thickness wounds on the dorsal side of mice created using a 5$\mathrm{mm}$ biopsy punch were treated with $\beta$-glucan-based hydrogel for 2 weeks. Standardized photographs of the wound site were taken at regular time intervals to calculate the percentage of wound closure. Tissues isolated from the wound area were subjected to histological examination and immunoblot analysis.

Results: $\beta$-Glucan-based hydrogel significantly accelerated the duration of wound healing and enhanced the development of skin appendages in the regenerated skin tissue. Increased expression of transforming growth factor- $\beta 3$ in the skin tissue isolated from the healed wound site indicated that skin regeneration rather than skin repair occurred, thereby minimizing cutaneous scarring. The expression level of cytokeratin 10 and cytokeratin 14 in the isolated skin tissue revealed that the wounds treated with hydrogel showed proper differentiation and proliferation of keratinocytes in the epidermal layer.

Innovation: Immunomodulating $\beta$-glucan (responsible for fighting infections at the wound site, and enhancing the migration and proliferation of keratinocytes and fibroblasts) in the form of a three-dimensional hydrogel membrane that retains a high water content (responsible for cooling and soothing effect around the wound site, thereby reducing pain) was prepared and analyzed for its effects on the cutaneous wound healing mechanism.

Conclusion: $\beta$-Glucan-based hydrogels are promising as wet wound dressings in the health care industry.

Keywords: antiscarring, wet dressing, $\beta$-glucan, immunomodulating, wound healing, hydrogel

\section{INTRODUCTION}

THE SKIN IS THE largest organ of the integumentary system, and acts as a protective barrier for the human body by providing electrolytic balance, heat regulation, and evaporation and microbial control, among other benefits. ${ }^{1}$ According to the World Health Or- ganization, skin injuries are expected to be the leading cause of death worldwide by 2020 , overwhelming other infectious diseases. ${ }^{2}$ Globally, $\sim 6$ million people had sought medical treatment annually for burns according to the World Burn Foundation until 2014. ${ }^{3}$ Burns, blisters,

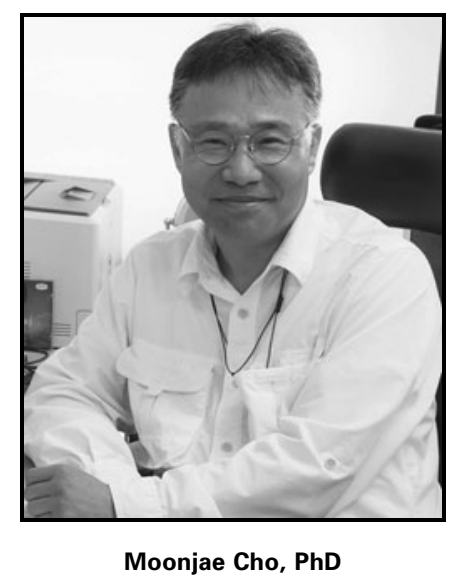

Submitted for publication August 31, 2018. Accepted in revised form November 15, 2018.

${ }^{*}$ Correspondence: Department of Biochemistry, School of Medicine, Jeju National University, Jeju, Republic of Korea (e-mail: moonjcho@jejunu.ac.kr or ymeekim3118@gmail.com). 
punctures, abrasions, contusions, and superficial injuries to the skin can result in trauma or septic shock, causing the skin to lose its ability to protect the human body. In addition, when a wound becomes "chronic" as a result of inflammation, hypertrophic scarring is more likely to occur. Treatment strategies for acute and chronic wounds arising from accidents, surgical injuries, burns, and ulcers include autologous skin transplant, sutures, staples, adhesive sealant, physiologic dressings, and administration of antibiotics. The treatment strategy plays a significant role in the outcome of the healing process, and lessening pain and discomfort experienced by the injured patients. Because of its low availability and scar formation potential, autologous skin transplant is less widely applied compared with dressing materials. ${ }^{4}$

After Yannas' group first developed dressings based on synthetic hydrogels modified with polymeric materials, researchers worldwide examined polymeric materials as biomedical devices. ${ }^{4} \mathrm{Hy}-$ drogels, which are three-dimensional polymeric materials, can retain high water content, prolonging exposure of the wound to a moist environment. This favors the migration and proliferation of fibroblasts during the proliferative phase of wound healing, leading to re-epithelialization at the wound site. ${ }^{5,6} \mathrm{~A}$ higher water content in a hydrogel improves its ability to cool, sooth, and reduce pain in the wound area. ${ }^{7}$ In addition, the humid conditions provided by the hydrogel aid in spontaneous detachment of dead tissue from the healthy tissue below. Although the moist environment provided by a hydrogel promotes faster wound healing, bacterial propagation can occur at the wound site. $\beta$-Glucan has antibacterial and antiviral properties, and may be useful for overcoming this limitaion. ${ }^{8}$

Recognized as pathogen-associated molecular patterns (PAMPs), $\beta$-glucans are a heterogeneous group of polysaccharides that are potent innate immune inducers. ${ }^{9,10}$ Through their ability to attract macrophages, neutrophils, and other immune cells, these water-soluble PAMPs fight infections at the wound site, and enhance the migration and proliferation of keratinocytes and fibroblasts, which are critical events in the complicated wound healing process. In addition, $\beta$-glucan shows antiinflammatory and antioxidant effects, which are essential for injury healing.

In this study, a hydrogel prepared from blends of immune-modulating $\beta$-glucan and poly (vinyl alcohol) (PVA) was developed using the solvent casting method, and its effect on wound healing was evaluated in detail. In addition, the ability of the developed PVA/ $\beta$-glucan hydrogel to act as a carrier for flavonoids was examined.

\section{CLINICAL PROBLEM ADDRESSED}

Traditional wound dressings such as pads, gauze, and cotton wool do not provide an appropriate bed for wound healing, and periodic dressing changes are tedious, and may disrupt the newly synthesized epidermal layer during healing. Further, scar development is likely when the skin under the healed area is subjected to severe tension or a dry environment. To overcome these limitations and provide a better environment for complete wound healing, researchers have focused on developing wound dressings that can stimulate cells participating in the dynamic wound healing process and have antiscarring effects.

\section{MATERIALS AND METHODS \\ Wound dressings}

The hydrogel was prepared by a solvent casting method, wherein cross-linked blends of polymer blends were poured onto a Petri dish, followed by atmospheric air drying. In brief, at a 1:1 volume ratio, 4\% (w/v) PVA (MW 89,000-98,000, 99+\% hydrolyzed) solution was mixed with $0.4 \% \beta$-glucan solution ([ $\beta$-1,6-branched- $\beta$-1,3-glucan, average MW, $3.5 \times 10^{6} \mathrm{Da}$ ] originated from Schizophyllum spp.) while stirring for $10 \mathrm{~min}$ at $60^{\circ} \mathrm{C}$. Glycerol and $1 \%$ glutaraldehyde were mixed into the solution at 1:25 and 1:10 volume ratios, respectively, and further stirred for $30 \mathrm{~min}$ to obtain a homogeneous solution. In the place of $\beta$-glucan, an equal volume of distilled water was added to the $4 \%$ PVA solution to obtain PVA hydrogel. Flavonoidincorporated hydrogels were prepared by adding a 1:1 molar concentration $(100 \mu \mathrm{M})$ of glycitin $\left(4^{\prime}-\right.$ hydroxy-6-methoxyisoflavone-7-D-glucoside) and $4^{\prime}, 6,7$-trimethoxyisoflavone (TMF) to the PVA $\beta$ glucan hydrogel solution before casting.

\section{Wound-dressing properties}

One of the crucial aspects of a wound treatment strategy is providing an appropriate bed for the healing process while clearing exudates from the wound surface, thereby increasing the rate of wound healing. ${ }^{11}$ These properties of an ideal dressing were analyzed by determining the water vapor transmission rate (WVTR) and swelling index of the topical hydrogel.

An ideal wound dressing prevents excessive dehydration at the wound site. The potential to transmit bodily fluid or wound exudates is reflected 
in the WVTR. Each hydrogel was cut into a circular shape and mounted on the mouth of a cylindrical bottle (diameter of $18 \mathrm{~mm}$ ) containing $30 \mathrm{~mL}$ of deionized water. After weighing $\left(\mathrm{W}_{\mathrm{i}}\right)$, this apparatus was incubated at $37^{\circ} \mathrm{C}$ for $24 \mathrm{~h}$. After $24 \mathrm{~h}$, the mass of the bottle was noted $\left(\mathrm{W}_{\mathrm{f}}\right)$. The WVTR of the hydrogel was determined as follows:
IX70 microscope, Tokyo, Japan, equipped with a digital camera).

Human keratinocytes (HaCaTs) were cultured at an initial density of $5 \times 10^{3}$ cells per well for $24 \mathrm{~h}$. After removing the growth medium, $200 \mu \mathrm{L}$ of the prepared hydrogel extracts were added. Cellular viability was then measured after $24 \mathrm{~h}$

WVTR $\left(\mathrm{g} / \mathrm{m}^{2}\right.$ day $)=\left(\mathrm{W}_{\mathrm{i}}-\mathrm{W}_{\mathrm{f}}\right) /$ area of the membrane under study.

The ability of the hydrogel to absorb wound exudates was analyzed by determining the swelling index of each hydrogel exposed to various solutions. To measure the swelling ratio of each hydrogel, the hydrogels were cut into $1 \times 1-\mathrm{cm}$ pieces, and then immersed in distilled water and $0.9 \%$ saline. In addition, the effect of $\mathrm{pH}$ on the waterholding capacity of the PVA/ $\beta$-glucan hydrogel was studied using buffers with different $\mathrm{pH}$ values $(\mathrm{pH}$ 4.0, 5.8, 7.0, 7.4, and 9.0). At specific time points, the immersed hydrogel pieces were removed carefully from the solutions and weighed after removing the adsorbed surface water using blotting paper. The following formula was used to calculate the swelling ratio:

$$
\text { Swelling ratio }=\left(\mathrm{W}_{\mathrm{t}} / \mathrm{W}_{\mathrm{i}}\right) \times 100,
$$

where $\mathrm{W}_{\mathrm{t}}$ and $\mathrm{W}_{\mathrm{i}}$ correspond to the weights of the sample at specific time points during immersion and weight of the sample before immersion, respectively.

\section{In vitro cytocompatibility studies}

The cytocompatibility of the hydrogels was investigated by observing the cellular morphology and viability of the dermal and epidermal layers. The experiments were performed using extracts of the hydrogel in Dulbecco's Modified Eagle's Medium (DMEM) as reported previously with slight modifications. ${ }^{12}$ First, $0.12 \mathrm{~g}$ of hydrogel was weighed and sterilized under ultraviolet light for a period of $30 \mathrm{~min}$, followed by immersion in $12 \mathrm{~mL}$ of DMEM for $24 \mathrm{~h}$ at $37^{\circ} \mathrm{C}$. After $24 \mathrm{~h}$, the medium was collected and sterilized using a $0.20-\mu \mathrm{m}$ filter membrane. The filtered hydrogel extract was used for in vitro studies. Cells cultured in growth medium were used as controls.

Primary human dermal fibroblasts were seeded at a density of $1 \times 10^{4}$ cells per well in a six-well plate. After allowing the cells to grow for $24 \mathrm{~h}$ at $37^{\circ} \mathrm{C}$ in $5 \% \mathrm{CO}_{2}$ incubator, the growth medium was exchanged for the hydrogel extract collected in the growth medium. After $48 \mathrm{~h}$, cell morphologies were observed under an optical microscope (Olympus using an MTT [3-(4,5-dimethylthiazol-2-yl)-2,5diphenyltetrazolium bromide] assay with six replicates.

\section{In vitro wound healing ability}

$\mathrm{HaCaT}$ cells were seeded into six-well cell culture plates at a cell density of $5 \times 10^{4}$ cells per well and grown in DMEM containing $10 \%$ fetal bovine serum for $24 \mathrm{~h}$. After $24 \mathrm{~h}$, a sterile pipette was used to make a scratch on the monolayer at the center of the well. The well was washed carefully with $1 \times$ phosphate-buffered saline, followed by incubation with PVA and PVA/ $\beta$-glucan hydrogel extract. At 0,24 , and $48 \mathrm{~h}$, the scratched area was photographed under an optical light microscope at $100 \times$ magnification. The migration rate for wound closure was calculated using ImageJ software (NIH, Bethesda, MD) based on the change in the margins of the scratch area.

\section{Cutaneous wound model-in vivo approach for wound healing}

Six-week-old Institute of Cancer Research mice were used for the experiment. All procedures were approved by the Animal Care and Use Committee, Jeju National University, Jeju, Republic of Korea. The mice were adapted to the laboratory animal room for 1 week at $23^{\circ} \mathrm{C} \pm 2^{\circ} \mathrm{C}$ with constant humidity $(55 \% \pm 15 \%)$ on a 12 -h light/12-h dark cycle. At the end of the adaptation period, the mice were randomly placed into five groups $(n=6$ for each group) as follows: negative control group (no treatment), test group (PVA hydrogel, PVA $\beta$-glucan hydrogel, flavonoid-incorporated PVA $/ \beta$-glucan hydrogel), and MeditouchH (positive control group) (Ildong Bioscience Co. Ltd., Gyeonggi-do, Republic of Korea). Elastic tape was used to hold the hydrogel to the wound. In brief, the dorsal side of each mouse was shaved, and a wound was created using a 5-mm punching tool. On days $0,4,7,10$, and 14 , the wound area was photographed using a digital camera (ruler was used to have the same field of view of the wound area) to determine the percentage of wound closure. The images were processed using ImageJ software to calculate the percentage 
of wound closure at each time point by analyzing the wound area left exposed.

\section{Histology}

To analyze skin regeneration, tissues collected on days 17 and 21 (three mice from each group) of the treatment period were subjected to histopathological staining. Tissues were isolated from the wound area fixed in $10 \%(\mathrm{v} / \mathrm{v})$ formalin solution for a minimum of 2 days, followed by embedding in paraffin wax. The embedded tissue samples were sectioned at $4-\mu \mathrm{m}$ thickness and stained for granulation tissue by hematoxylin and eosin (H\&E) staining. The stained samples were visualized under an Olympus BX51 microscope at different magnifications. From the obtained image, epidermal thickness was measured with ImageJ software by considering the thickness at ten different locations along the re-epithelialized skin tissue on days 17 and 21. Localization of cytokeratin proteins in the skin tissue isolated on day 17 was carried out by immunohistochemical analysis using the primary antibodies: cytokeratin 10 (sc-53252) and cytokeratin 14 (sc-17104).

\section{Protein expression analysis}

Proteins were extracted from the skin tissue isolated on day 17 using T-per buffer (Thermo Scientific, Waltham, MA) as reported previously. ${ }^{13}$ Equivolume samples containing $20 \mu \mathrm{g}$ of protein per lane were used to examine protein expression by western blotting. Samples were tested for expression of the following proteins (Catalog No. and concentration of the primary antibody used is given in parentheses): cytokeratin 10 (K10) (sc-53252; 1:500), cytokeratin 14 (K14) (sc-17104; 1:4000), transforming growth factor- $\beta 3$ (TGF- $\beta 3$ ) (sc$166861 ; 1: 1000)$, and $\beta$-actin (sc-47778; $1: 1000)$ as the loading control. All primary antibodies were purchased from Santa Cruz Biotechnology (Dallas, TX). The blots were developed using a chemiluminescence ECL kit (LPS solution, Daejeon, Korea). The bands were then quantified using ImageJ software (https://imagej.nih.gov/ij/).

\section{Statistical analysis}

All results were expressed as the mean \pm standard error. The wound area left exposed for the indicated time and thickness of the epidermal layer at days 17 and 21 based on H\&E staining and protein expression analysis were quantified using Image J software. Graphs were plotted using Microsoft Excel. Significant differences between test groups were determined using independent $t$-test (between two groups) and one-way analysis of variance (for more than two groups) with a cutoff significance of $p<0.05$.

\section{RESULTS}

The PVA and PVA/ $\beta$-glucan hydrogels fabricated in this study (Fig. 1A) showed WVTRs of $2930.80 \pm 101.78$ and $2427.83 \pm 36.58 \mathrm{~g} / \mathrm{m}^{2} /$ day, respectively (Fig. 1B). The swelling ratio of PVA/ $\beta$-glucan hydrogel reached a maximum in distilled water than that observed in saline solution when immersed in the respective medium (Fig. 1C, D) with $p$-value $<0.1$. In addition, the swelling ratio was higher for PVA $/ \beta$-glucan hydrogel than for the PVA hydrogel. Analysis of the impact of $\mathrm{pH}$ on the fluid uptake capacity (given in Fig. 1E) indicated that the PVA/ $\beta$-glucan hydrogel immersed in alkaline medium ( $\mathrm{pH}$ of 9) showed significant swelling of more than fourfold its original weight.

The effect of hydrogel extract on cellular attachment and morphology of the human dermal fibroblasts was observed using microscopic images. The morphology of cells grown under the influence of PVA/ $\beta$-glucan hydrogel extract showed no detectable changes (Fig. 2A), with similar or slightly enhanced growth rates compared with cells grown in DMEM; in contrast, cells grown under the influence of PVA hydrogel showed few cells with slightly malformed degenerative structures. According to ISO 10993-5:2009, any material that shows cellular viability $>70 \%$ of that of the control is considered nontoxic with good cytocompatibility. Quantitative cell viability results from the MTT assay (Fig. 2B) showed that in the PVA and PVA/ $\beta$-glucan hydrogel groups, cell viability was $>80 \%$ of that of tissue-culture-grade polystyrene dishes-grown keratinocytes (statistically significant), suggesting that these hydrogels are excellent materials for biomedical applications. Further, the in vitro scratch wound healing assay showed that HaCaT cells cultured in the PVA $/ \beta$ glucan hydrogel extract had a faster migration rate than cells grown in PVA hydrogel extract medium (Fig. 2C).

Full-thickness wounds created on the dorsal sides of mice were treated with PVA hydrogel, PVA/ $\beta$-glucan hydrogel, flavonoid-incorporated (1:1 molar concentration of glycitin and TMF) PVA/ $\beta$-glucan hydrogel, MeditouchH (positive control), and not treated. As shown in Fig. 3A, B, wound closure was faster in the PVA $/ \beta$-glucan hydrogeltreated mice group, with $>75 \%$ of the wound areas closed on day 10, followed by the positive control, PVA hydrogel, and nontreated control groups. Further, the flavonoid-incorporated PVA/ $\beta$-glucan hydrogel showed significantly ( $p$-value $<0.01$ ) faster wound closure (20\%) compared with the nontreated control at day 4 postinjury. While scabs appeared starting on day 4 of the treatment period in the PVA/ 

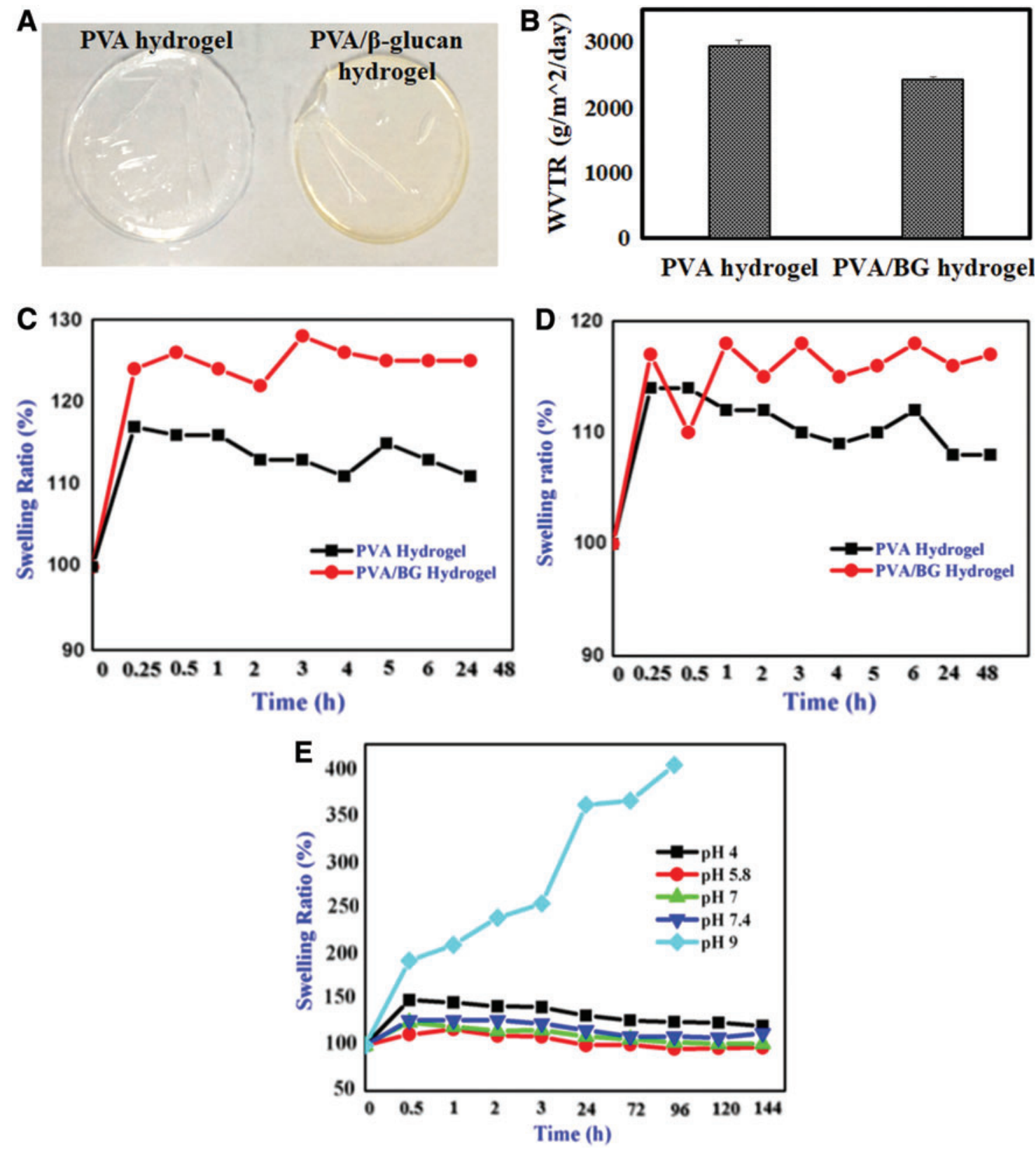

Figure 1. (A) Photograph of the developed PVA and PVA/ $\beta$-glucan hydrogel. (B) Moisture permeation capacities of the hydrogels $(n=3)$. The results are shown as the mean \pm standard error. The fluid absorption capacity of PVA and PVA/ $\beta$-glucan hydrogels in (C) distilled water and (D) $0.9 \%$ saline solution ( $p$ value $<0.1$ ) and $(E)$ swelling ratio of the PVA/ $\beta$-glucan hydrogel immersed in phosphate-buffered saline solution at different $\mathrm{pH}$. The values were significant at $p<0.05$. PVA, poly (vinyl alcohol). Color images are available online.

$\beta$-glucan hydrogel-treated mice, the scabs disappeared with the onset of re-epithelialization on day 10. In contrast, the remaining groups showed scabs beyond day 10. Healing was indicated as redpinkish skin on the wound bed.

Histological analysis (Fig. 4A) showed that mice treated with PVA/ $\beta$-glucan and flavonoidincorporated PVA/ $\beta$-glucan hydrogel onto the cutaneous wound were in the remodeling phase of the healing process with well-developed skin appendages, glandular substances, capillary vessel formation, better granulation, hierarchical arrangement of dermal layers, and re-epithelialization similar to that of normal skin. Flavonoid incorporation enhanced wound healing during the initial phase of the process and promoted proper skin regeneration around the wound site.

On days 17-21 of the remodeling phase postinjury (Fig. 4B), the thickness of the epidermal layer in the test groups approached that of the normal skin, with the $\mathrm{PVA} / \beta$-glucan hydrogeltreated group showing the fastest rate, and the 


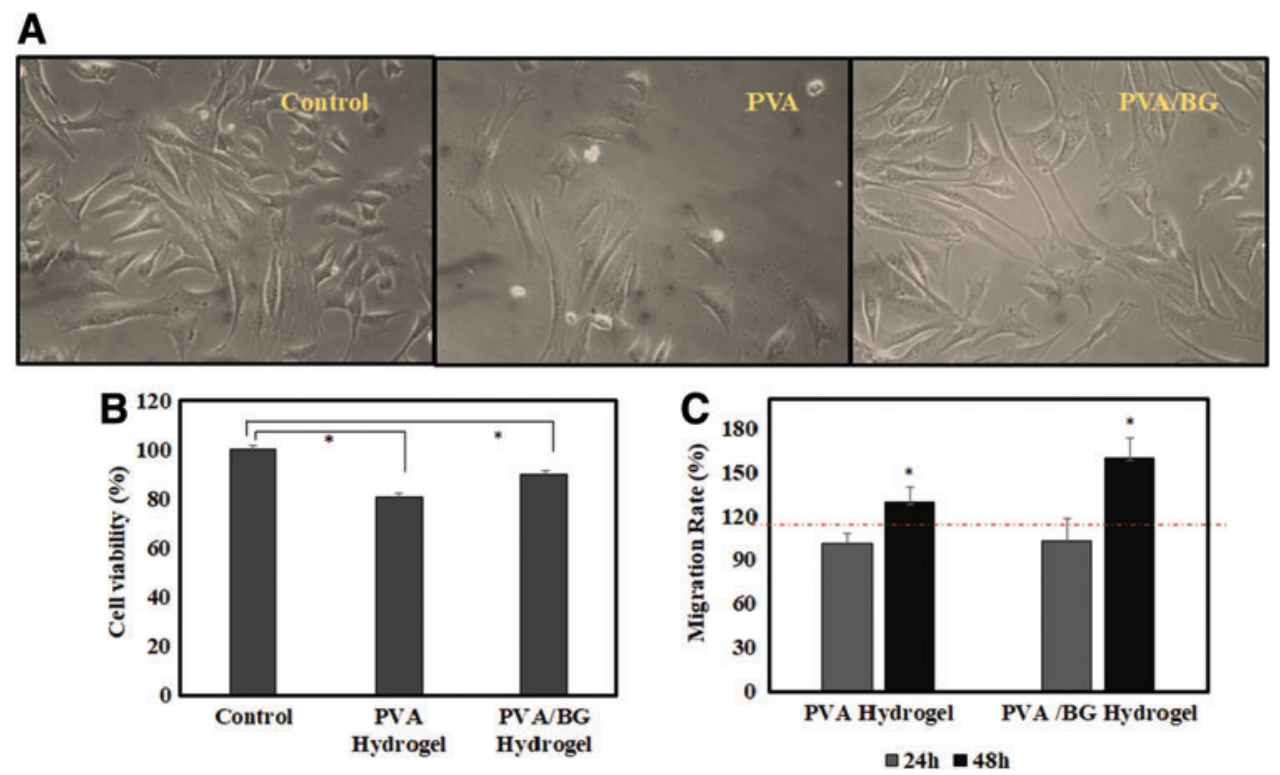

Figure 2. Cellular compatibility of the hydrogels. (A) Morphology of human dermal fibroblast cells cultured in DMEM (control) and hydrogel extract in DMEM (magnification $=200 \times$ ) are shown. (B) Cell viability of HaCaT keratinocytes grown in hydrogel extract for $24 \mathrm{~h}$ as determined by MTT assay $(n=6)$. (C) $I n$ vitro wound closure attributed to the effects of hydrogel extract on keratinocyte cells $(n=2)$. The red dashed line indicates the migration rate under the influence of hydrogel extract compared with that of the control. *Represents $p<0.05$ relative to that of control and was considered significant. DMEM, Dulbecco's Modified Eagle's medium; HaCaTs, human keratinocytes. Color images are available online.

results were significant. Protein expression analysis of the isolated skin tissue on or around the wound site on day 17 postinjury revealed enhanced translational levels of K10, K14, and TGF- $\beta 3$ proteins in mouse groups treated with $\mathrm{PVA} / \beta$-glucan hydrogel (Fig. 5A). Localization of keratin proteins in the isolated skin tissue was observed through immunohistochemical analysis (Fig. 5B, C), and it was found out that K10 and K14 were circumscribed to the suprabasal and basal layer of the skin tissue, respectively.

\section{DISCUSSION}

In this study, we developed a bioactive and biocompatible hydrogel that promotes cutaneous wound healing while fighting infections at the wound site, promoting cellular migration to increase the rate of wound closure, and providing a moist bed for highly complex wound healing dynamics.

The water vapor diffusion ability of the hydrogel must be optimal; high or low values are associated with excessive dehydration or leakage of exudate from the wound site, respectively. The WVTR of healthy skin is $204 \pm 12 \mathrm{~g} / \mathrm{m}^{2} /$ day, whereas in traumatized skin, these values are $279 \pm 26$ and $5138 \pm 202 \mathrm{~g} / \mathrm{m}^{2} /$ day for burned skin and granulating wounds, respectively. ${ }^{14}$ For the PVA/ $\beta$-glucan hydrogel, the WVTR was in the suggested range $\left(2000-2500 \mathrm{~g} / \mathrm{m}^{2} /\right.$ day) of ideal wound dressings, thereby providing requisite moisture without risking wound bed dehydration.

The ability of the hydrogel to absorb wound exudates was analyzed by determining the swelling index of each hydrogel in various solutions. For the same volume of immersion solution, the degree of swelling was higher for hydrogels immersed in the distilled water than in saline solution. While studying the effects of $\mathrm{pH}$ on the swelling behavior of the PVA/ $\beta$-glucan hydrogel, the degrees of swelling with solutions at $\mathrm{pH} 4.0$ and 7.4 were higher than those in solutions at $\mathrm{pH} 5.8$ and 7. However, under alkaline conditions ( $\mathrm{pH}$ 9.0), the swelling ratio was drastically increased (up to $350 \%$ of its original weight). As infected wound beds and exudate fluid have $\mathrm{pH}$ values ranging from 7.5 to 8.9, the higher swelling ratio of the PVA/ $\beta$-glucan hydrogel under alkaline conditions makes it a better candidate for removing oozes from the wound bed while maintaining a moist environment. ${ }^{15}$

As a potent macrophage stimulator, $\beta$-glucan plays a vital role in the dynamic wound healing process by releasing wound growth factors through macrophage stimulation. ${ }^{16}$ Healing efficiency evaluated at the microscopic level using H\&E staining revealed abnormal deposition of the epidermis into the dermis layer in the control groups on day 17 . In contrast, the PVA/ $\beta$-glucan hydrogel- 
A
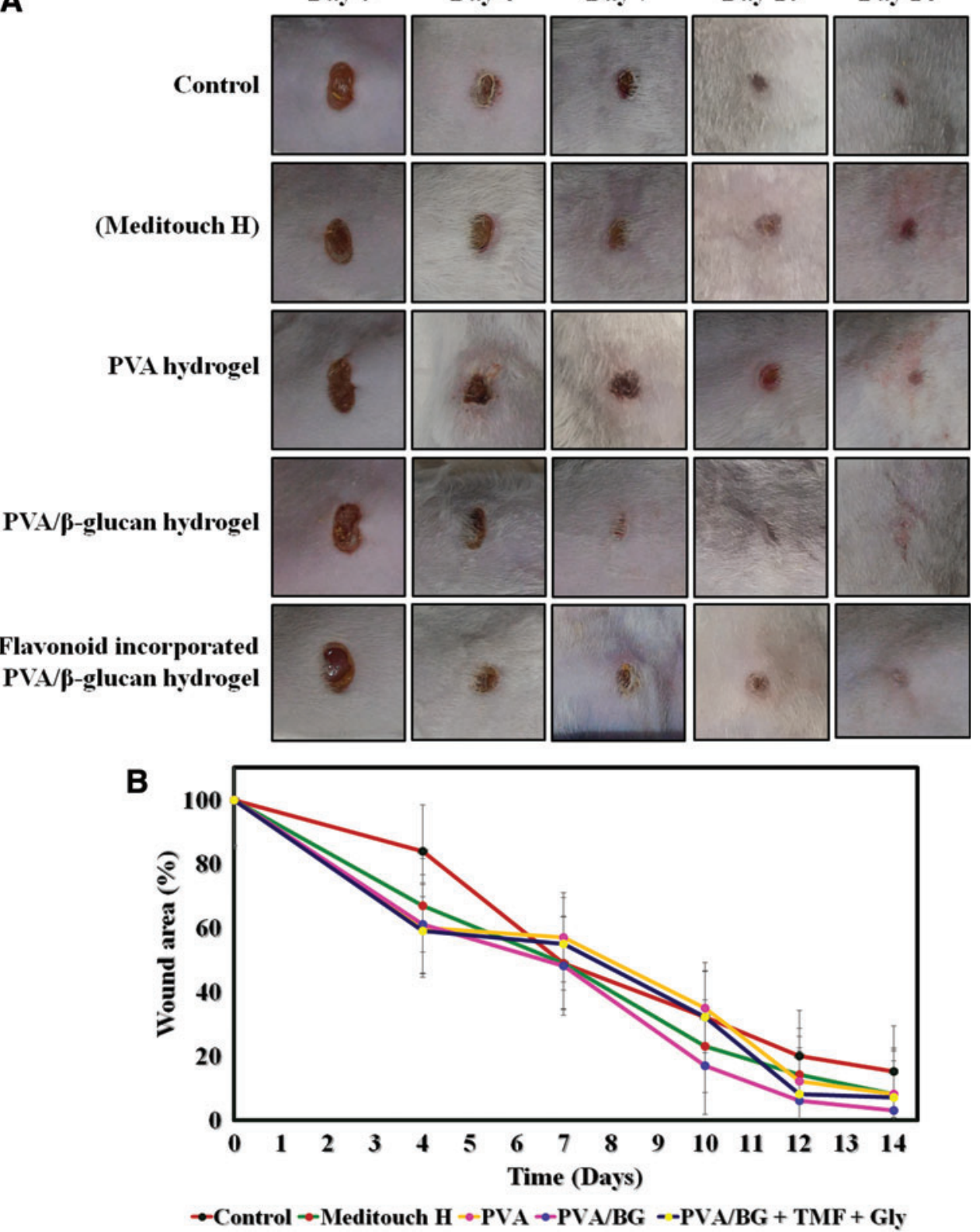

Figure 3. Effects of the hydrogels on in vivo wound healing evaluated using a mouse wound model. (A) Standardized photographs of wound healing with different dressings at various times are shown. (B) Open wound areas on the indicated days after injury $(n=6)$ are represented graphically. The result is presented as the mean \pm standard error. Color images are available online.

treated group showed smooth epidermal tissue with signs of better granulation, hierarchical arrangement of the dermal layers, and skin appendages. This result indicates that $\beta$-glucanincorporated PVA hydrogels have high clinical potential as a wound-dressing biomaterial. On days 17-21 postinjury, the thickness of the epidermal layer in the PVA/ $\beta$-glucan hydrogel test groups approached that of the normal skin with better efficiency.

In immunoblot analysis, the translational expression levels of K10, K14, and TGF- $\beta 3$ proteins in the skin tissue isolated on or around the wound site were found to be highest in the PVA $/ \beta$-glucan hydrogel-treated group. The increase in expression of protein K10, a keratinocyte differentiation marker, in the $\mathrm{PVA} / \beta$-glucan hydrogel-treated group agrees with the results of the epidermal thickness study. During the remodeling phase, keratinocytes covering the wound bed began to get flattened, matured, and differentiated, forming a thin, multilayered stratified epithelial layer. ${ }^{17}$ The rapid approach of the epidermal thickness in the PVA $/ \beta$ glucan hydrogel-treated group to that of the normal 

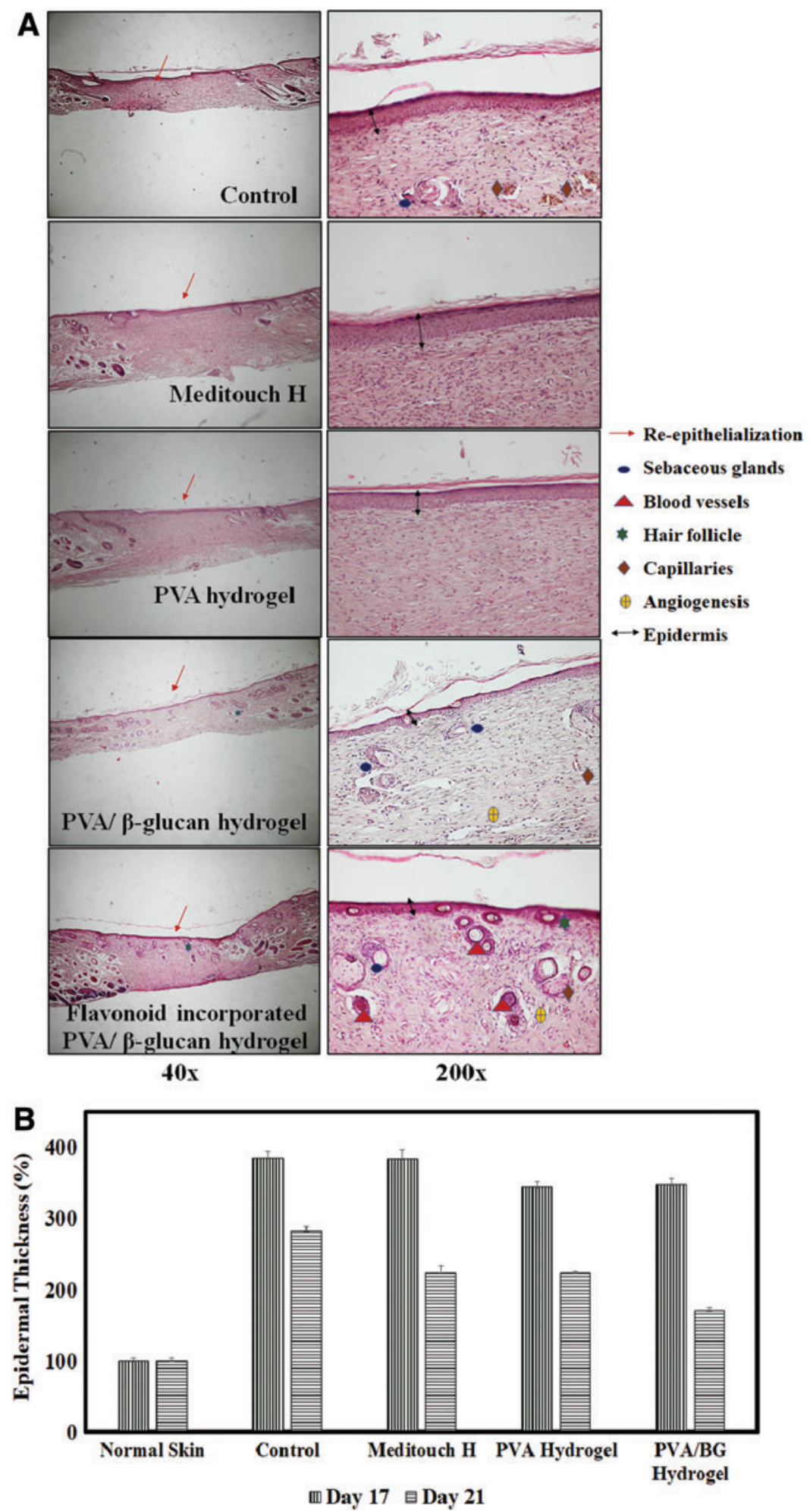

Figure 4. (A) Pathological examination using H\&E staining of regenerated skin tissue at the wound site treated with different dressings (magnification: 40 and $200 \times$ ). (B) Epidermal thickness of the skin tissue isolated from the healed wound area on days 17 and 21. The results were significant at $p<0.05$. Skin tissues were isolated from the wound site on days 17 and 21, and the effects of the hydrogels on re-epithelialization with proper granulation were examined. The epithelial thickness was calculated using H\&E-stained tissue samples (magnification: 100×) using ImageJ software. H\&E, hematoxylin and eosin. Color images are available online. 
A
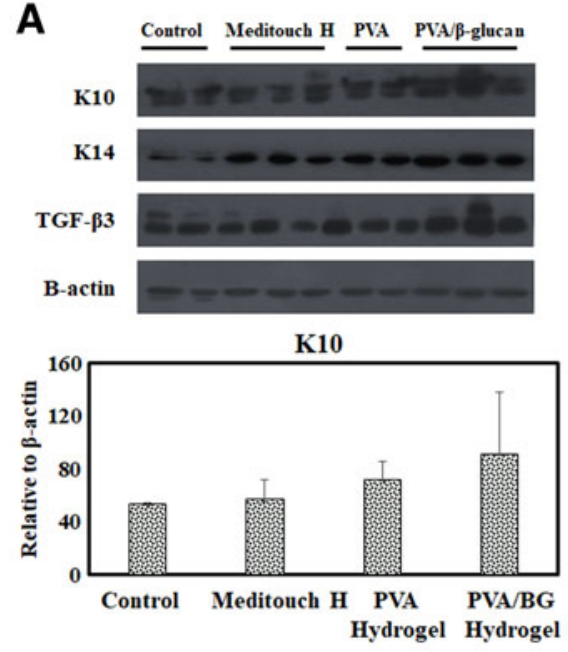

B
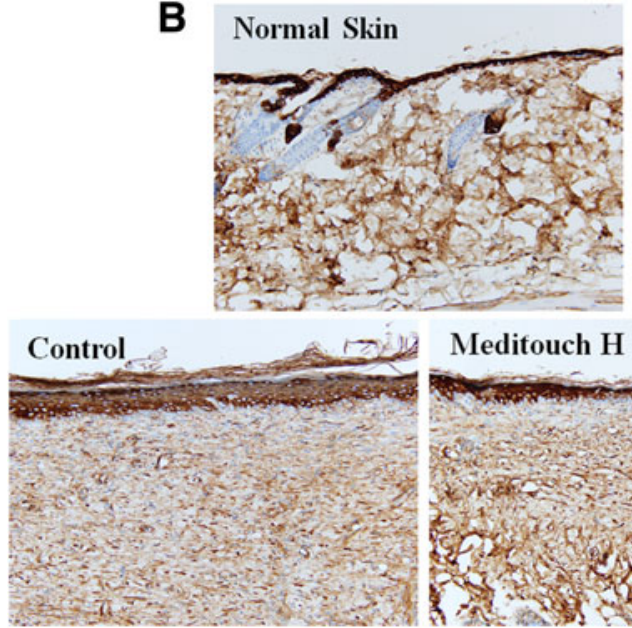

PVA hydrogel

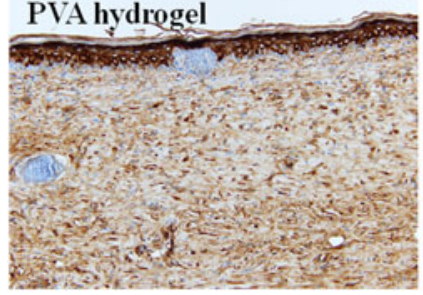

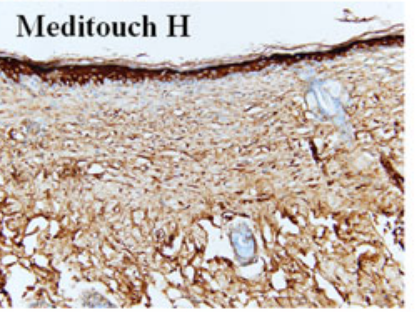

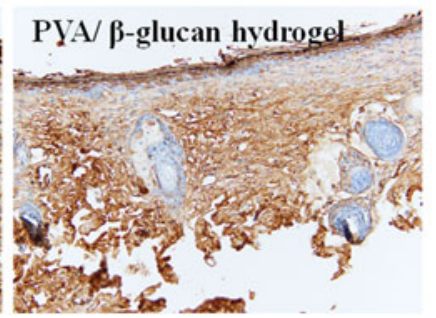

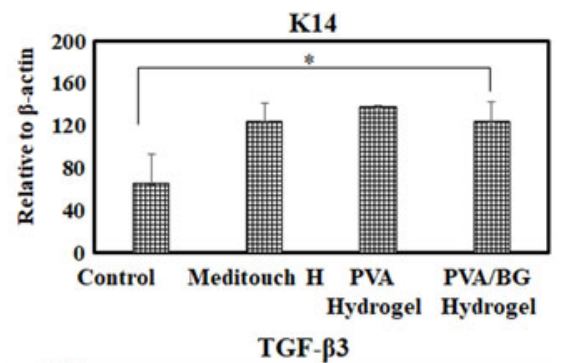

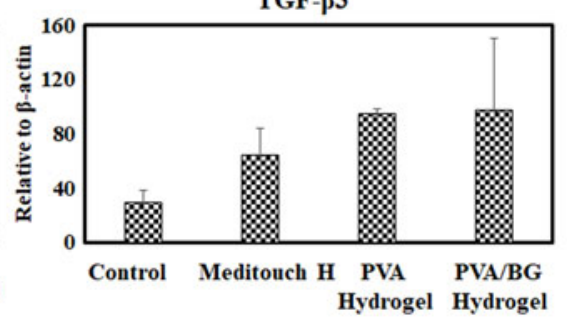

C Normal Skin
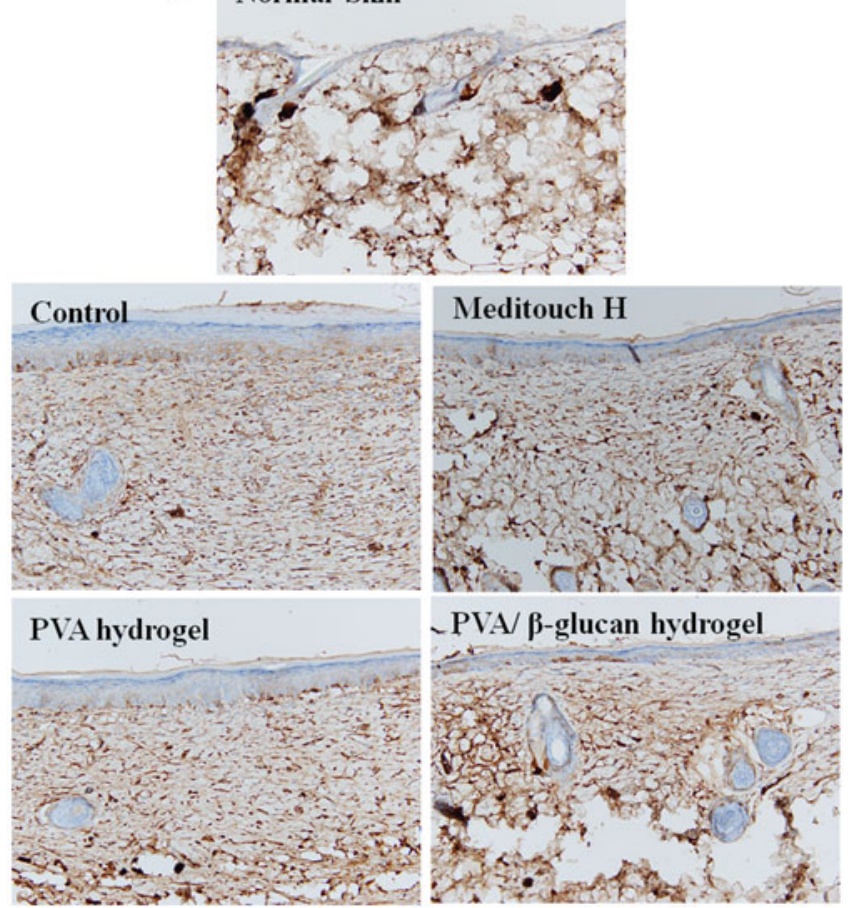

-PVA/ $\beta$-glucan hydrogel

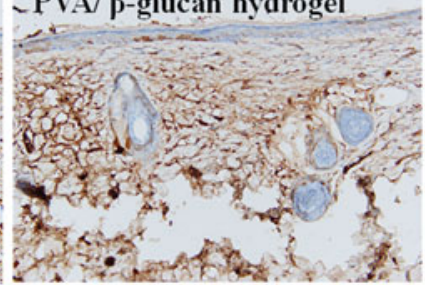

Figure 5. (A) Protein immunoblot assay of the skin tissue isolated from the wound site postinjury on day 17 to determine the expression of K10 (terminal keratinocyte differentiation marker), K14 (basal keratinocyte proliferation marker), and TGF- $\beta 3$ (antiscar marker). The blots were processed with ImageJ software, and the corresponding values are plotted on a graph using Excel. * Represents $p<0.05$ relative to that of control and was considered significant. Localization of keratin proteins through immunohistochemical analysis of the skin tissue isolated on day 17 (B) cytokeratin 10 and (C) cytokeratin 14 . TGF- $\beta 3$, transforming growth factor $\beta$-3. Color images are available online.

skin indicates that the $\beta$-glucan-incorporated hydrogel has a good wound healing capacity with proper epidermal reorganization. Expression of the proliferation marker K14, found in the stratum basal of the epidermal layer, was similar in all groups except for in the untreated control group. Proper coverage of the wound bed with basal keratinocytes was marked by keratinocyte proliferation factors such as keratin 5 and 14, which promote reorganization of the underlying epidermal and dermal layers. ${ }^{18}$ The lower K14 expression in the control group suggests that reepithelialization was not well developed, posing the risk of scar development. This agrees with the reduced expression of TGF- $\beta 3$, an antiscarring mammalian isoform of TGF- $\beta$, by coordinating the remodeling phase of the skin wound healing process. When TGF- $\beta 3$ is highly expressed, skin regeneration is greatly facilitated rather than skin repair, thereby minimizing cu- 
taneous scarring. ${ }^{19-21}$ Based on the proper differentiation and proliferation of keratinocytes in the epidermal layer (as indicated by increased expression of K10 and K14), along with minimal or no scar formation (as indicated by increased expression of TGF- $\beta 3$ ) and proper development of skin appendages (as indicated by H\&E staining), the $\beta$-glucan-incorporated hydrogel showed good potential for clinical applications in wound healing.

Isoflavones, TMF, and glycitin showed synergistic effects on wound healing at a 1:1 ratio. A cutaneous wound model treated with these flavonoid-incorporated PVA/ $\beta$-glucan hydrogels showed enhanced wound closure (a difference of $20 \%$ wound closure compared with the control on day 4 postinjury) during the initial stage. Thus, flavonoid incorporation plays an important role in wound treatment support by increasing the healing rate.

\section{Summary}

In this study, a hydrogel prepared from immunomodulating $\beta$-glucan was synthesized as a wet-dressing material. The developed hydrogel exhibited good water-holding capability and stability as well as high cytocompatibility of $>80 \%$. An in vitro wound healing study showed that the epidermal cells migrated 1.5-fold faster under the PVA/ $\beta$-glucan hydrogel extract than with the control. The hydrogel significantly promoted skin wound healing, not only in vitro but also in vivo, with better remodeling of newly formed skin tissue. In addition, the hydrogel can act as a carrier for flavonoids to enhance the development of skin appendages in the regenerated skin and increase the rate of wound closure during the initial phase of wound healing. Protein immunoblot assays against antiscarring (TGF- $\beta 3$ ), proliferation (K14), and differentiation (K10) markers along with histopathological study suggested that the hydrogel is a promising candidate as a wound care management strategy.

\section{INNOVATION}

Immunomodulating $\beta$-glucan (responsible for fighting infections at the wound site and enhancing the migration and proliferation of keratinocytes and fibroblasts, thereby aiding in faster wound closure with proper development of skin appendages) in the form of a three-dimensional hydrogel membrane that retains high water content (responsible for cooling and soothing effect around the wound site, thereby reducing pain) was employed for cutaneous wound healing.

\section{ACKNOWLEDGMENTS AND FUNDING SOURCES}

This research was supported by the Korea Industrial Complex Corporation through the project FOSTERING THE INDUSTRIAL CLUSTER (No. RKK17008).

\section{AUTHOR DISCLOSURE AND GHOST WRITING}

The authors declare no conflicts of interests. The listed authors wrote this article, and no ghostwriters were used for article writing.

\section{ABOUT THE AUTHORS}

Moonjae Cho, PhD, is a professor in the Department of Biochemistry, School of Medicine, Jeju National University, Republic of Korea. He also acted as the director of the Institute of Medical Science, Jeju National University from 2016. He served as an exchange professor in UC Davis, United States in 2005-2006. He worked as a postdoctoral fellow at Oklahoma State University, USA. Seung In Choi is a team leader in Medical Business at the Pharmaceutical Research Institute, Quegen Biotech Co. Ltd., Seoul. Young Mee Kim, PhD, is currently a research professor at the Department of Biochemistry, School of Medicine, Jeju National University. Changlim Hyun is an associate professor in the Department of Pathology, School of Medicine, Jeju National University, Republic of Korea. He is specialized in the area of surgical pathology and cytopathology. Karthika Muthuramalingam, MSc, is a secondyear doctoral student in the Department of Biochemistry, School of Medicine, Jeju National University. She completed her Master's in Nano Science and Technology, and her undergraduate studies in the field of biotechnology. 


\section{REFERENCES}

1. Chen $X$, Zhang $M$, Wang $X$, et al. Peptide-modified chitosan hydrogels promote skin wound healing by enhancing wound angiogenesis and inhibiting inflammation. Am J Transl Res 2017;9:2352-2362.

2. National Institutes of Health. Burns and Traumatic Injury. Bethesda, MD: National Institute of General Medical Sciences, 2016:1-2.

3. Wounds International. International Best Practice Guidelines: effective Skin and wound management of non-complex burns 2014. https://www .woundsinternational.com/resources/details/bestpractice-guidelines-effective-skin-and-woundmanagement-in-non-complex-burns (last accessed January 3, 2019).

4. Kamoun EA, Chen X, Mohy Eldin MS, Kenawy ERS. Crosslinked poly(vinyl alcohol) hydrogels for wound dressing applications: a review of remarkably blended polymers. Arabian $\mathrm{J}$ Chem 2015;8:1-14.

5. Gong CY, Wu OJ, Wang YJ, et al. A biodegradable hydrogel system containing curcumin encapsulated in micelles for cutaneous wound healing. Biomaterials 2013;34:6377-6387.

6. Madaghiele M, Sannino A, Ambrosio L, Demitri C. Polymeric hydrogels for burn wound care: advanced skin wound dressings and regenerative templates. Burns Trauma 2014;2:153.

7. Zhao X, Wu H, Guo B, et al. Antibacterial antioxidant electroactive injectable hydrogel as self-healing wound dressing with hemostasis and adhesiveness for cutaneous wound healing. Biomaterials 2017;122:34-47.

8. Chamidah A, Hardoko H, Prihanto AA. Antibacterial activities of $\beta$-glucan (laminaran) against gram-negative and gram-positive bacteria. AIP Conference Proc 2017;1844:020011-1-020011-7.

9. Grip J, Engstad RE, Skjæveland I, et al. Sprayable carbopol hydrogel with soluble beta-1,3/1,6glucan as an active ingredient for wound healingdevelopment and in-vivo evaluation. Eur J Pharm Sci 2017;107:24-31.

10. Gwon HJ, Lim YM, Park JS, Nho YC. Evaluation of radiation synthesized $\beta$-glucan hydrogel wound dressing using rat models. World Academy of Science, Engineering and Technology, International Science Index 60. Int J Med Health Biomed Bioeng Pharm Eng 2011;5:684-687.

11. El-Kased RF, Amer Rl, Attia D, Elmazar MM. Honey-based hydrogel: in vitro and comparative in vivo evaluation for burn wound healing. Sci Rep 2017:7:1-11.

12. Zhang L, Ma Y, Pan X, Chen S, Zhuang H, Wang S. A composite hydrogel of chitosan/heparin/poly $(\gamma$ glutamic acid) loaded with superoxide dismutase for wound healing. Carbohydr Polym 2018;180: 168-174.

13. Muthuramalingam K, Ho J, You K, Jeon J, Rho S. Effects of sea horse (Hippocampus abdominalis) -derived protein hydrolysate on skeletal muscle development. J Appl Biol Chem 2017;60: 373-381.

14. Mi FL, Shyu SS, Wu YB, Lee ST, Shyong JY, Huang RN. Fabrication and characterization of a sponge-like asymmetric chitosan membrane as a wound dressing. Biomaterials 2001;22:165173.

15. Liu L, Li X, Nagao M, Elias AL, Narain R, Chung HJ. A pH-Indicating colorimetric tough hydrogel patch towards applications in a substrate for smart wound dressings. Polymers 2017;9:558.

16. Cerci C, Yildirim M, Ceyhan M, Bozkurt S, Doguc D, Gokicimen A. The effects of topical and systemic beta glucan administration on wound healing impaired by corticosteroids. Wounds 2008;20:341-346.

17. ter Horst B, Chouhan G, Moiemen NS, Grover LM. Advances in keratinocyte delivery in burn wound care. Adv Drug Deliv Rev 2018;123:18-32.

18. Pastar I, Stojadinovic 0, Yin NC, et al. Epithelialization in wound healing: a comprehensive review. Adv Wound Care 2014;3:445-464.

19. Pakyari M, Farrokhi A, Maharlooei MK, Ghahary A. Critical role of transforming growth factor beta in different phases of wound healing. Adv Wound Care 2013;2:215-224.

20. Le M, Naridze R, Morrison J, et al. Transforming growth factor beta 3 is required for excisional wound repair in vivo. PLoS One 2012;7:1-10.

21. Penn JW, Grobbelaar AO, Rolfe KJ. The role of the TGF- $\beta$ family in wound healing, burns and scarring: a review. Int J Burns Trauma 2012;2:1828.

\footnotetext{
Abbreviations and Acronyms

DMEM = Dulbecco's Modified Eagle's medium

$\mathrm{H} \& \mathrm{E}=$ hematoxylin and eosin

HaCaTs = human keratinocytes

PAMP $=$ pathogen-associated molecular patterns

$\mathrm{PVA}=$ poly (vinyl alcohol)

TGF- $\beta 3=$ transforming growth factor $-\beta 3$

TMF $=4^{\prime}, 6,7$-Trimethoxyisoflavone

WVTR $=$ water vapor transmission rate
} 